\title{
Magnetic resonance features of sinonasal melanotic mucosal melanoma
}

\author{
Cosimo Nardi, ${ }^{1}$ Chiara Vignoli, ${ }^{1}$ Margherita Vannucchi, ${ }^{\oplus 2}$ Michele Pietragalla ${ }^{1}$
}

Department of Experimental and Clinical Biomedical Sciences, Radiodiagnostic Unit n. 2, University of Florence - Azienda OspedalieroUniversitaria Careggi, Florence, Italy

${ }^{2}$ Department of Surgery and Translational Medicine, Division of Pathological Anatomy, University of Florence - Azienda Ospedaliero-Universitaria Careggi, Florence, Italy

\section{Correspondence to}

Dr Michele Pietragalla, michelepietragalla@hotmail.it

Accepted 8 July 2019

\section{SUMMARY}

A 67-year-old man presented to ear, nose and throat department complaining of nasal congestion and recurrent epistaxis for 5 months. Nasal endoscopy revealed a pigmented polyp obstructing the right nasal cavity. MRI with contrast agent showed a right nasal cavity polypoid mass with hyper signal intensity (SI) both in non-enhanced T1-w and diffusion imaging, marked hypo SI in T2-w sequences and avidly contrast enhancement characterised by rapid wash-in without significant wash-out on dynamic perfusion imaging. Histological specimen showed epithelioid and spindle cells with focal intense pigmentations and immunohistochemical features compatible with primary melanotic sinonasal mucosal melanoma (SNM). As melanotic SNM shows MRI pathognomonic high nonenhanced T1-W SI, this case underlines the crucial role of MRI not only in assessing the local tumour extension/ recurrence but also in increasing the diagnostic confidence of detecting melanotic SNM. Thus, MRI should be always performed in case of clinical-endoscopic suspicion of SNM.

\section{BACKGROUND}

Primary malignant sinonasal mucosal melanoma (SNM) is a rare and heavily aggressive sinonasal neoplasm. It represents $<4 \%$ of all head and neck

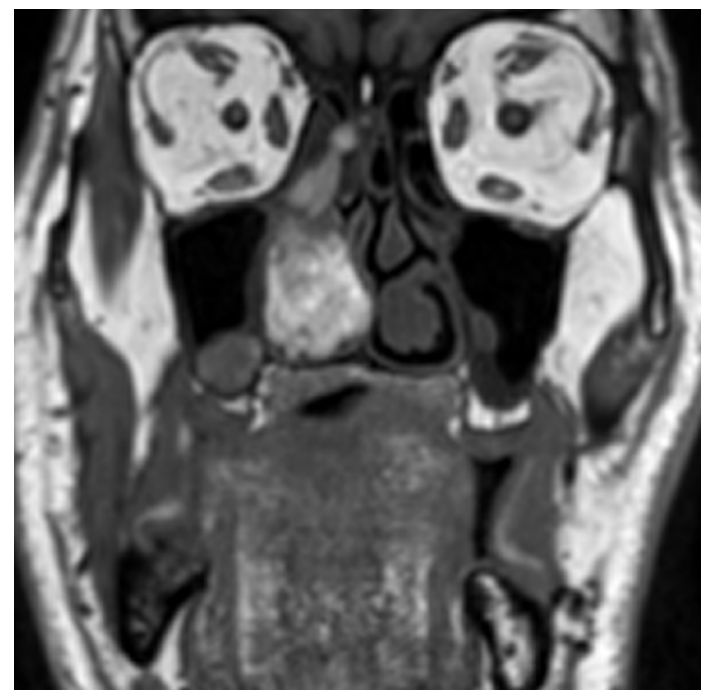

Figure 1 T1-w non-contrast enhanced coronal MRI. Polypoid lesion in the right nasal cavity with heterogeneous hyper signal intensity involves the inferior and middle meatus.

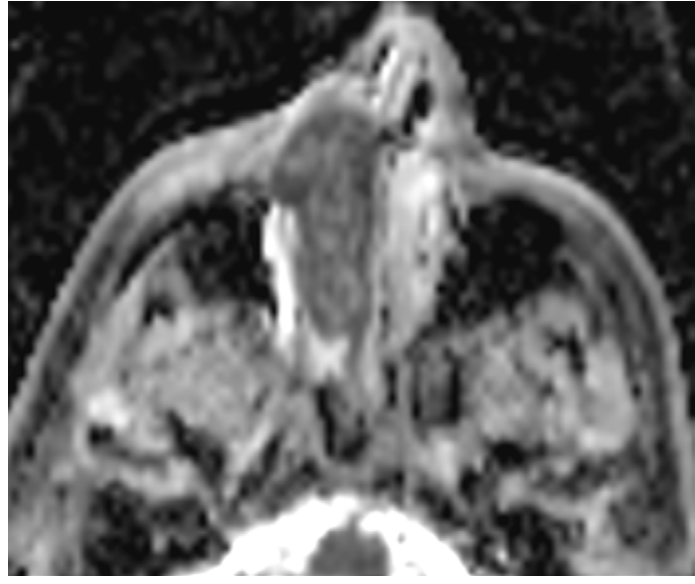

Figure 2 Apparent diffusion coefficient (ADC) map shows a polypoid lesion in the right nasal cavity with low ADC values, a typical feature of SNM. Bilateral inflammatory mucosal changes with high $A D C$ values are also present.

cancer, $55 \%$ of all head and neck melanoma and $0.3 \%-2 \%$ of all melanomas (annual incidence rate 0.5 per million). ${ }^{1} \mathrm{SNM}$ mostly arises from melanocytes of the mucosa of the nasal cavity $(50 \%$ of cases) and paranasal sinuses (20\%). ${ }^{2-4}$ The average age of onset for SNM is 65 years (M:F 3:2). ${ }^{5}$ The etiopathogenetic is related to the cigarette smoking, formaldehyde and genetic alterations, whereas the solar exposure does not seem to play a key role. ${ }^{4}$ The primary sites of involvement are the nasal septum, the nasal lateral wall and the inferior nasal concha. ${ }^{1}$ It is necessary to exclude that the tumour is the secondary manifestation of cutaneous or uveal melanomas. ${ }^{2}$ Furthermore, symptoms are no specific (epistaxis, nasal obstruction and headache) and therefore the diagnosis is often delayed. ${ }^{6} \mathrm{MRI}$ with contrast agent is the technique of choice in the study of monolateral sinonasal polypoid lesion since it distinguishes between solid lesions and inflammatory mucosal changes. As melanin has strongly hyperintense and hypointense signal intensity (SI) respect to the muscle in non-enhanced T1-w and T2-w sequences, respectively, ${ }^{3}$ MRI can be an important diagnostic tool in the diagnosis of melanotic SNM. MRI with diffusion-weighted imaging (DwI) and dynamic contrast enhancement imaging (DCE-PwI) can provide useful functional information in the characterisation of SNM. Moreover, MRI plays a basic role in the local-regional staging of malignant neoplasms with a higher diagnostic 


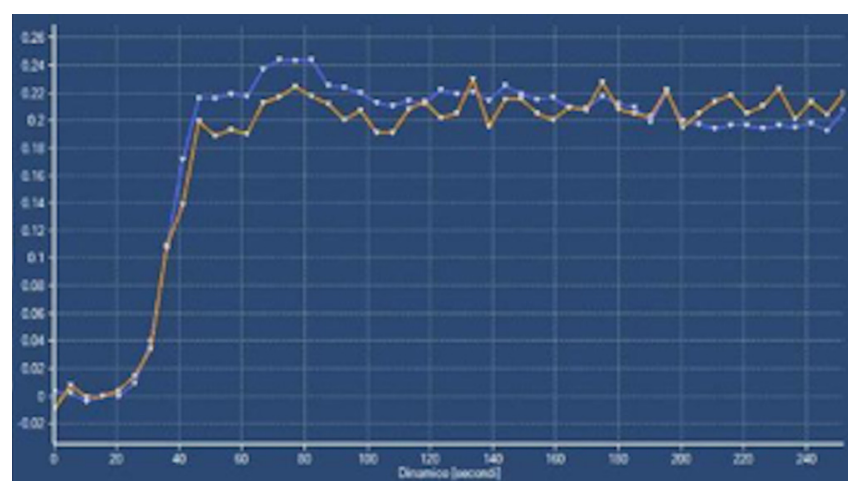

Figure 3 Dynamic contrast enhancement perfusion-weighted imaging time-intensity curve shows a rapid wash-in without significant washout, a finding highly suspicious for malignancy.

accuracy than CT in the assessment of dural, anterior skull base and orbit invasion. ${ }^{78}$

\section{CASE PRESENTATION}

A 67-year-old patient presented to ear, nose and throat department complaining of nasal congestion and recurrent epistaxis for 5 months. Anticoagulant therapy, previous head and neck surgery, and history of tumour were not referred in anamnesis. The clinical examination showed a nasal profile deformation.

\section{INVESTIGATIONS}

Baseline biochemical and haematological investigations showed no abnormalities. The nasal endoscopy revealed a pigmented, ulcerated and friable polypoid mass that completely obstructed the right nasal cavity. MRI showed a mass occupying the right middle and inferior meatus characterised by hyper SI both in T1-w (figure 1) and DwI sequences (figure 2), and marked hypo $\mathrm{SI}$ in T2-w sequences. After intravenous contrast agent injection, the lesion showed marked enhancement with rapid wash-in and without significant wash-out on DCE-PwI time/intensity curve (figure 3). Therefore, an endoscopic biopsy was performed. Histological specimen of the lesion showed epithelioid and spindle cells with a focal and intense melanin pigmentation and immunohistochemical features compatible with primary sinonasal melanotic mucosal melanoma (figures 4 and 5).

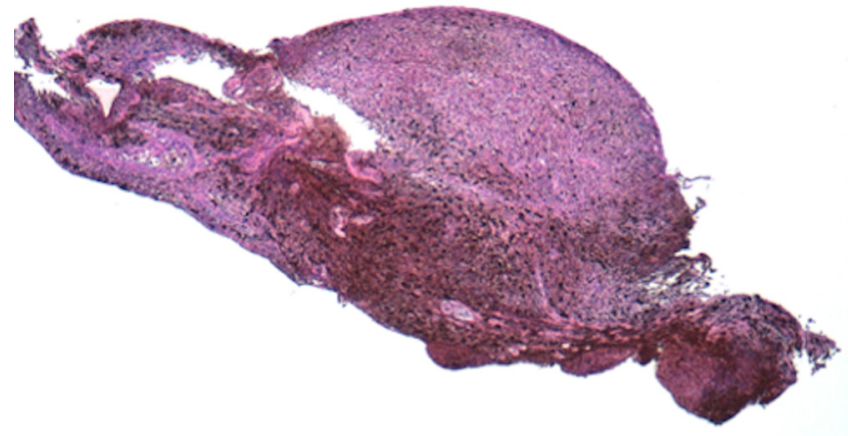

Figure 4 Histological specimen shows an aggregate of epithelioid and spindle cells with focal and intense pigmentation (magnification, 5x).

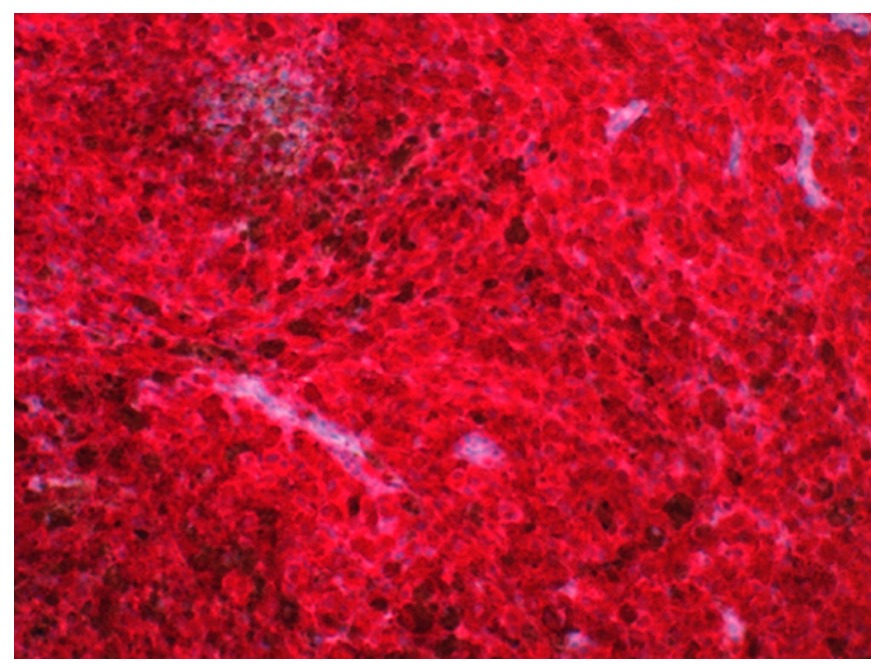

Figure 5 Positivity for Melanoma-associated antigen recognized by $\mathrm{T}$ cells (MART-1) immunostaining (magnification, 20x).

\section{TREATMENT}

The patient underwent the surgical removal of the nasal mass and subsequent cycles of radiotherapy and chemotherapy.

\section{OUTCOME AND FOLLOW-UP}

In the staging phase, fluorodeoxyglucose (18F-FDG) positron emission tomography (PET) and total body CT with contrast agent showed a pancreatic metastasis (figure 6). Early follow-up with

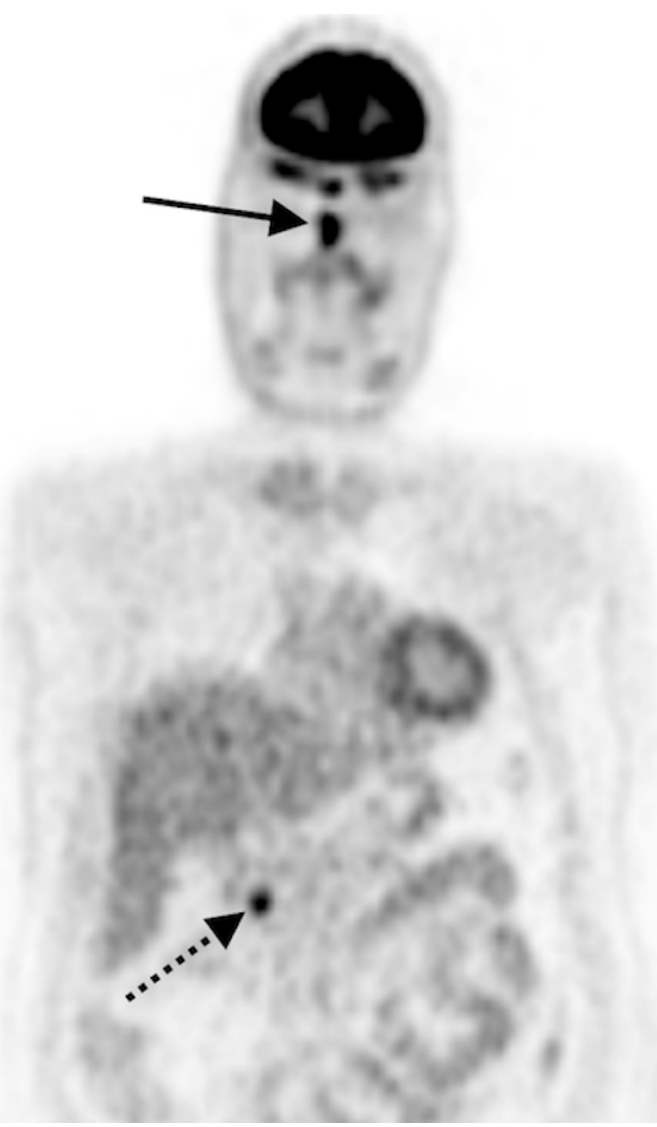

Figure 6 18F-FDG positron emission tomography detects significant accumulation of the tracer in the nasal cavity (black arrow) and pancreatic head (black dotted arrow). 


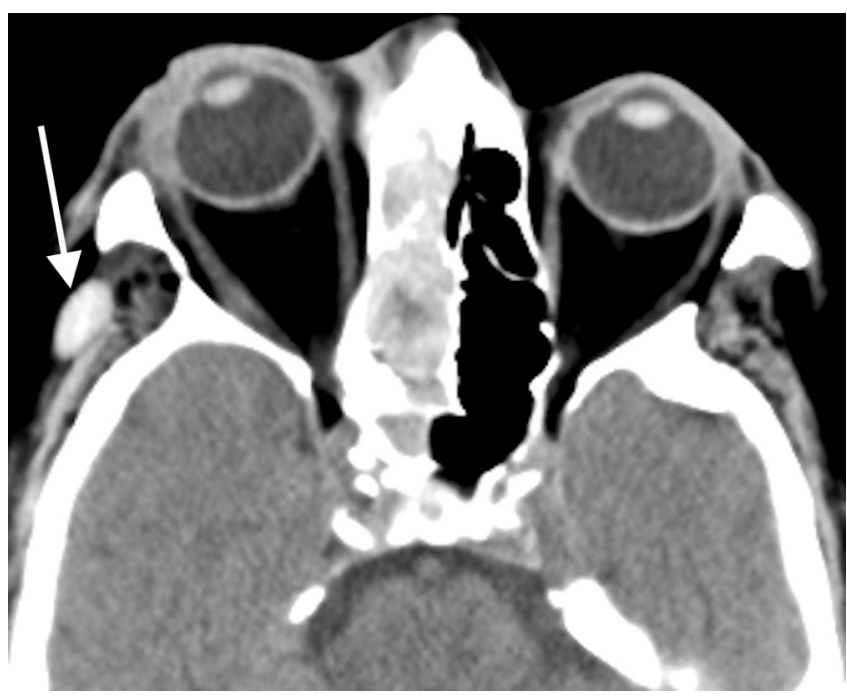

Figure 7 Enhanced axial CT scan points out the massive local sinonasal recurrence and a metastasis in the right temporal muscle (white arrow).

total body CT showed both local and distant relapses of the disease. Metastases in the right temporal muscle (figure 7), bladder dome and lung appeared. Therefore, the immunological therapy was implemented. Nevertheless, the next CT scan revealed a further rapid progression of the disease with an increase of lung nodules and the appearance of liver metastases. The patient died after 3 months.

\section{DISCUSSION}

MRI plays a crucial role in the diagnosis, characterisation and local-regional staging of SNM. Therefore, MRI should be always performed if SNM is suspected from clinical-endoscopic examination. MRI features of SNM are variable, due to varying amounts of melanin, with up to one-third of cases being amelanotic. ${ }^{9}$ Melanin has strongly hyperintense SI respect to the muscle in non-enhanced T1-w and hypointense SI in T2-w sequences, ${ }^{3}$ thus melanotic SNM can be easily detected with MRI. On the contrary, amelanotic melanoma appears isointense to muscle in non-enhanced T1-w sequences. ${ }^{6}$ In SNM, DwI shows diffusion restriction with a high signal in images at high b-value (b8001000) and low values in apparent diffusion coefficient (ADC) map, that prove the typical behaviour of malignant lesions (figure 2). ${ }^{10}$ DCE-PwI examines microvascular characteristics of tumour tissue giving information about benignity or malignancy. Malignant tumours are expected to have a rapid and vividly enhancement, despite benign lesions tend to have a progressive enhancing pattern. ${ }^{11} 12$ Tumour invasion of the orbits, base of the skull, intracranial and nasopharynx is common. T2-w sequences on the coronal plane allow to evaluate the erosion of the orbit inferior wall, lamina papyracea and ethmoidal cribriform plate, whereas isotropic-isovolumetric fat saturation sequences with contrast enhanced help to identify the infiltration of the dura mater and/or anterior cranial fossa. ${ }^{7813}$ Cerebral oedema and leptomeningeal/cerebral impregnation in direct contact with the tumour are indicative of brain involvement. ${ }^{14}$ Distant metastases are common too. ${ }^{15}$ 18F-FDG PET-CT scan FDG-PET/CT has been shown to be useful in evaluating residual or recurrent $\mathrm{SNM} .{ }^{16} \mathrm{SNM}$ is a very aggressive tumour with a poor prognosis with up to $64 \%$ recurrence 1 year after surgery ${ }^{17}$ and overall 5 -year survival $<25 \% .{ }^{18}$ In conclusion, SNM is a rare entity and the nasal cavity is the most common site of origin. MRI can provide significant information in the diagnosis and local staging of SNM. High MRI SI on non-contrast enhanced T1-w and DwI sequences, low ADC values and rapid wash-in without noteworthy wash-out on DCE-PwI are very suggestive of melanotic SNM. PET-CT examination is mandatory for the assessment of distant metastasis.

\section{Learning points}

- MRI with contrast agent is the technique of choice in the study of monolateral sinonasal polypoid lesion since it distinguishes between solid lesions and inflammatory mucosal changes.

- MRI plays a crucial role in the diagnosis and local-regional staging of sinonasal mucosal melanoma (SNM) and, therefore, it should be always performed in case of clinicalendoscopic suspicion.

- Melanoma of the head and neck region is a rare entity and the nasal cavity is its most common site of origin. Melanotic SNM shows pathognomonic MRI features, especially a high signal intensity (SI) on non-contrast enhanced T1-w and diffusion-weighted imaging sequences, low $\mathrm{SI}$ on $\mathrm{T} 2-\mathrm{w}$, and marked enhancement after intravenous contrast agent injection.

- 18F-FDG positron emission tomography-CT examination is mandatory in patients with SNM both for diagnosis and follow-up to screen for distal metastases and local recurrences since they are common events and often associated with poor prognosis.

Contributors CN: paper's structure and review. CV: data collection. MV: histological images. MP: paper's structure and review.

Funding The authors have not declared a specific grant for this research from any funding agency in the public, commercial or not-for-profit sectors.

Competing interests None declared.

Patient consent for publication Obtained

Provenance and peer review Not commissioned; externally peer reviewed.

\section{REFERENCES}

1 Clifton N, Harrison L, Bradley PJ, et al. Malignant melanoma of nasal cavity and paranasal sinuses: report of 24 patients and literature review. J Laryngol Otol 2011:125:479-85.

2 Azoury SC, Epidemiology LJR, Factors R. Prevention, and Early Detection of Melanoma. Surg Clin North Am 2014;94:945-62.

3 Eggesbø HB. Imaging of sinonasal tumours. Cancer Imaging 2012;12:152.

4 Gilain L, Houette A, Montalban A, et al. Mucosal melanoma of the nasal cavity and paranasal sinuses. Eur Ann Otorhinolaryngol Head Neck Dis 2014;131:365-9.

5 Mochel MC, Duncan LM, Piris A, et al. Primary mucosal melanoma of the sinonasal tract: a clinicopathologic and immunohistochemical study of thirty-two cases. Head Neck Pathol 2015:9:236-43.

6 Alves IS, Berriel LG, Alves RT, et al. Sinonasal Melanoma: A Case Report and Literature Review. Case Rep Oncol Med 2017;2017:1-6.

7 Borges A, Coiteiro D, Santos JR, et al. Using magnetic resonance for the imaging of dural invasion by head and neck tumors. Imaging Med 2010;2:593-602.

8 Turri-Zanoni M, Lambertoni A, Margherini S, et al. Multidisciplinary treatment algorithm for the management of sinonasal cancers with orbital invasion: $A$ retrospective study. Head Neck 2019.

9 Nguyen B. Sinonasal malignant melanoma with diffuse metastasis: F-18 FDG PET/CT imaging. Radiology Case Reports 2008;03:3.

10 Ascierto PA, Accorona R, Botti G, et al. Mucosal melanoma of the head and neck. Crit Rev Oncol Hematol 2017;112:136-52.

11 Bernstein JM, Homer JJ, West CM. Dynamic contrast-enhanced magnetic resonance imaging biomarkers in head and neck cancer: potential to guide treatment? A systematic review. Oral Oncol 2014;50:963-70.

12 Gaddikeri S, Gaddikeri RS, Tailor T, et al. Dynamic contrast-enhanced mr imaging in head and neck cancer: Techniques and clinical applications. AJNR Am J Neuroradiol 2016:37:1-8.

13 lannetti G, Valentini V, Rinna C, et al. Ethmoido-orbital tumors: our experience. J Craniofac Surg 2005;16:1085-91. 
14 Maroldi R, Ravanelli M, Borghesi A, et al. Paranasal sinus imaging. Eur J Radiol 2008:66:372-86.

15 O'Regan K, Breen M, Ramaiya N, et al. Metastatic mucosal melanoma: imaging patterns of metastasis and recurrence. Cancer Imaging 2013;13:626-32.

16 Haerle SK, Soyka MB, Fischer DR, et al. The value of 18F-FDG-PET/CT imaging for sinonasal malignant melanoma. Eur Arch Otorhinolaryngol 2012;269:127-33.

Copyright 2019 BMJ Publishing Group. All rights reserved. For permission to reuse any of this content visit https://www.bmj.com/company/products-services/rights-and-licensing/permissions/

BMJ Case Report Fellows may re-use this article for personal use and teaching without any further permission.

Become a Fellow of BMJ Case Reports today and you can:

- Submit as many cases as you like

- Enjoy fast sympathetic peer review and rapid publication of accepted articles

- Access all the published articles

Re-use any of the published material for personal use and teaching without further permission

Customer Service

If you have any further queries about your subscription, please contact our customer services team on +44 (0) 2071111105 or via email at support@bmj.com.

Visit casereports.bmj.com for more articles like this and to become a Fellow
17 Yoshioka H, Kamada T, Kandatsu S, et al. MRI of mucosal malignant melanoma of the head and neck. J Comput Assist Tomogr 1998;22:492-7.

18 Gal TJ, Silver N, Huang B. Demographics and treatment trends in sinonasal mucosal melanoma. Laryngoscope 2011;121:n/a-2033. 